\title{
Experimental designs for evaluation of genetic variability and selection of ancient grapevine varieties: a simulation study
}

\author{
E Gonçalves ${ }^{1}$, A St.Aubyn ${ }^{2,3}$ and A Martins ${ }^{1}$ \\ ${ }^{1}$ Centro de Botânica Aplicada à Agricultura (CBAA), Instituto Superior de Agronomia, Technical University of Lisbon, Tapada da Ajuda, \\ Lisboa, Portugal; ${ }^{2}$ Instituto Superior de Agronomia, Technical University of Lisbon, Tapada da Ajuda, Lisboa, Portugal and ${ }^{3}$ Centro de \\ Investigação em Turismo, Inovação e Serviços (CITIS), Universidade Lusíada de Lisboa, Rua da Junqueira, Lisboa, Portugal
}

\begin{abstract}
Classical methodologies for grapevine selection used in the vine-growing world are generally based on comparisons among a small number of clones. This does not take advantage of the entire genetic variability within ancient varieties, and therefore limits selection challenges. Using the general principles of plant breeding and of quantitative genetics, we propose new breeding strategies, focussed on conservation and quantification of genetic variability by performing a cycle of mass genotypic selection prior to clonal selection. To exploit a sufficiently large amount of genetic variability, initial selection trials must be generally very large. The use of experimental designs adequate for those field trials has been intensively recommended for
\end{abstract}

numerous species. However, their use in initial trials of grapevines has not been studied. With the aim of identifying the most suitable experimental designs for quantification of genetic variability and selection of ancient varieties, a study was carried out to assess through simulation the comparative efficiency of various experimental designs (randomized complete block design, $\alpha$ design and row-column (RC) design). The results indicated a greater efficiency for $\alpha$ and $\mathrm{RC}$ designs, enabling more precise estimates of genotypic variance, greater precision in the prediction of genetic gain and consequently greater efficiency in genotypic mass selection. Heredity (2010) 104, 552-562; doi:10.1038/hdy.2009.153; published online 11 November 2009

Keywords: grapevine; genetic variability; genetic selection; experimental design; simulation

\section{Introduction}

Grapevine is a very socially and economically important crop in many countries worldwide. It is thought that the ancient grapevine varieties have resulted from the domestication of individual wild plants, subsequently multiplied by vegetative propagation throughout centuries and millennia until the present time. As such, one variety would have been a single homogeneous clone at the beginning, but the effects of recurrent somatic mutations, and of other factors of variation, would have transformed it into a vast group of genotypes, with some morphological homogeneity, yet with differences in many quantitative characteristics of agronomical and technological interest (yield, sugar, acidity of the must and many others). Consequently, high genetic variation was generated within varieties along their evolutionary history.

Field experimentation with perennial plants is comparatively difficult and the selection methodology of grapevine varieties used in the vine-growing world in the last 50-100 years emphasizes the sanitary side of the

Correspondence: $\operatorname{Dr}$ E Gonçalves, Centro de Botânica Aplicada à Agricultura, Instituto Superior de Agronomia, Technical University of Lisbon, Tapada da Ajuda, Lisboa 1349-017, Portugal.

E-mail: elsagoncalves@isa.utl.pt

Received 29 June 2008; revised 18 September 2009; accepted 9 October 2009; published online 11 November 2009 selection process based on virus diagnosis (OIV, 1991) but neglects the potential of genetic variation.

However, understanding the entire variability existent within a variety and its distribution across the different regions and countries where it is grown is a very important matter because this will allow new views on history of agriculture and people (based on the relation between variability and evolutionary age of populations), a more efficient recognition and preservation of genetic resources (slowing down genetic erosion) as well as higher genetic gains through selection. As a consequence, our selection strategy in Portugal is mainly focussed on the knowledge about genetic variability (quantification, geographic distribution) through a method composed of three phases: (i) sampling variability in different regions where the variety is grown (hundreds of mother plants in ancient vineyards); (ii) planting a large field trial with the sampled plants (each one multiplied by vegetative propagation originating a clone); this phase has the objective of quantifying the genetic variability of the most important traits (typically yield, but also sugar content of the must, acidity and antocyanes) and of carrying out mass genotypic selection (selecting a group of clones); (iii) establishment of multienvironmental trials with the selected group in phase 2, with the aim of clonal selection. This paper refers to the second stage of this methodology.

To quantify the genetic variation within a variety and to perform efficient selection, through quantitative 
genetics as well as other methods, it is necessary to plant a very large field trial (normally from 200 to 400 clones). Only large trials contain a representative sample of all the variation within the variety across the different regions where it is grown. The fact that grapevine is perennial and the field trials are maintained for many years, allows us to make various genotypic mass selections, responding to future environmental alterations as well as to ever changing consumer demands. As a result, large field trials are in fact at the cutting edge of a new strategy for the evaluation and the usage of grapevine genetic resources.

However, the field trial above referred would cover an unusually large area (from 0.75 to $1.5 \mathrm{ha}$ ) which, by itself, causes large environmental variation. Therefore, the importance of experimental design in this type of trial is crucial to reach the objectives above referred successfully.

After Fisher (1935) introduced randomized complete block (RCB) designs, Yates (1936, 1940) described balanced incomplete block designs for the first time, including balanced square lattice designs. Since then, many variants on these designs have appeared. Although this group of designs is very large, we will restrict ourselves to only the most relevant for working with a high number of treatments, as frequently happens with initial trials of grapevine selection. Of note are $\alpha$ designs (Patterson and Williams, 1976), which constitute a particular class of generalized lattice designs, rowcolumn (RC) designs (Williams and John, 1989), and which correspond to groups of more complex latin square designs, t-latinized designs (John and Williams, 1998) and resolvable spatial RC designs (Williams et al., 2006). In sum, it can be said that $\alpha$ designs are resolvable designs and are recommended whenever the number of treatments is large. For these block designs there is no limitation on block size. For resolvable RC designs the plots in each replicate are arranged in rows and columns. A spatial resolvable RC design takes into account the separation of different treatments in rows and columns. For a latinized design the replicates are contiguous and form long blocks (or columns) of plots (for more details see the references above mentioned and Whitaker et al., 2007).

Many algorithms have been developed to build these designs, suitable for trials of more than 100 treatments (Patterson and Williams, 1976; Williams, 1985; Nguyen, 1994; Nguyen, 1997; Whitaker et al., 2007), as well as statistical tools to assess their efficiency (John and Whitaker, 2000).

The use of these experimental designs has been intensively recommended and discussed for decades. In the agricultural field, studies have reported greater effectiveness of balanced incomplete block and $\alpha$ designs compared to RCB designs (Patterson et al., 1978; Patterson and Silvey, 1980; Patterson and Hunter, 1983; Kempton et al., 1994; Yau, 1997; Qiao et al., 2000), and in the area of forestry similar results have been obtained, for example by Fu et al. (1998, 1999) and Gezan et al. (2006).

Although all these contributions are important, their use in initial trials of grapevines has not been studied. In this article we compare through simulation several of the aforementioned experimental designs (RCB, $\alpha$ and RC designs), with the aim of identifying those most suitable for quantifying and using the genetic variability under various different conditions: different field layouts, different population sizes, different levels of genetic variability of yield in the population, different levels of environmental variance. Through the simulations we attempted to clarify the effects of different experimental designs on the control of spatial variation, on the accuracy and precision of the estimates for genetic variance and on the prediction of genetic gain.

\section{Materials and methods}

\section{Simulation procedure}

Yield data were simulated because this trait has a general interest in all selection programmes and it is currently used for quantification of genetic variability under field conditions.

The simulated yield data were generated according to the model

$$
y_{i l m}=\mu+u_{g_{i}}+\varepsilon_{i l m}+\eta_{i l m}
$$

where $y_{i l m}$ is the observed yield located in the $l$ th column and $m$ th row, generated as the sum of the overall mean of the population $(\mu)$ with the genotypic effect of clone $g_{i}$ $\left(u_{g_{i}}\right)$ and the errors associated with the observation $y_{i l m}$, spatially dependent $\left(\varepsilon_{i l m}\right)$ and independent $\left(\eta_{i l m}\right)$.

The parametric values were established such that generated data showed a good agreement with the actual trials for grapevine selection. Thus, populations with an overall mean $(\mu)$ of $3 \mathrm{~kg}$ per plant were used. The genotypic effects were assumed independent and identically distributed (iid) random variables, with normal distribution with mean 0 and variance $\sigma_{g}^{2}$ $\left(u_{g_{i}} \sim N\left(0, \sigma_{g}^{2}\right)\right)$. Two values of genotypic variance were considered, $\sigma_{g}^{2}=0.2025$ and $\sigma_{g}^{2}=0.81$, corresponding, respectively, to populations with a smaller and larger genetic variation (Martins, 2007). Populations of 100, 200 and 300 genotypes were simulated, with both levels of variability. These populations were generated using SAS code, version 9.1 (SAS Institute, 2003), RANNOR function (100 simulations for each population type).

In this simulation study, the option for a high value for the error variance component $\left(\sigma_{e}^{2}\right)$ is justified because of the fact that we are working with a perennial plant that is influenced by many environmental factors, grown in poor and heterogeneous soils and requiring intensive management, which causes a high level of errors associated with the observations. Thus, according to what is observed in large grapevine field trials (around 70 in Portugal), two values of error variance were considered, $\sigma_{e}^{2}=1$ and 3 , corresponding respectively to level 1 (the most frequent) and level 2 of environmental variation. In respect to spatial variability, in these trials the percentage of spatially independent variation is usually around $60 \%$, but can oscillate between 50 and $70 \%$ of the total error variance (values obtained from current yield data analysis of 70 grapevine initial selection trials and also supported by Gonçalves et al., 2007). In this study, we assumed that $60 \%$ of the total error variance is attributable to the spatially independent variance and $40 \%$ to the spatially dependent variance. As a consequence, two error components 
were considered: an iid normal component $\eta_{\text {ilmm }}$, defined as $\eta_{i l m} \sim N\left(0, \sigma_{\eta}^{2}\right)$, and a spatially dependent component $\varepsilon_{i l m}$, defined as $N\left(0, \sigma_{\varepsilon}^{2} f\right)$, with

$f=\left(\exp \left(-1 / \theta_{\text {row }}\right)\right)^{\left\|h_{a b}\right\|_{\text {row }}}\left(\exp \left(-1 / \theta_{\text {col }}\right)\right)^{\left\|h_{a b}\right\|_{\text {col }}}$.

This is an anisotropic exponential model, where $\left\|h_{a b}\right\|_{\text {row }}=\left\|s_{a}-s_{b}\right\|_{\text {row }}$ is the Euclidian distance between the centre of the plot located at $S_{\mathrm{a}}$ and the centre of the plot located at $S_{\mathrm{b}}$ in row direction, $\left\|h_{a b}\right\|_{\text {col }}=\left\|s_{a}-s_{b}\right\|_{\text {col }}$ is the Euclidian distance between the centre of the plot located at $S_{a}$ and the centre of the plot located at $S_{b}$ in column direction and parameters $\theta_{\text {row }}$ and $\theta_{\mathrm{col}}$ are related to the ranges of correlation in row and column directions, respectively. It should be pointed out that under the exponential model the correlation reaches 0 only asymptotically. Therefore, for this model is used the term practical range $\left(3 \theta_{\text {row }}, 3 \theta_{\text {col }}\right)$ for specifying the distance where the covariance is reduced to $5 \%$ of the $\sigma_{\varepsilon}^{2}$, that is, the distance where the level of correlation is regarded as approximately 0 (Littell et al., 2006).

They were established values for the parameters $\theta_{\text {row }}$ and $\theta_{\text {col }}$ of 20 and $15 \mathrm{~m}$, respectively (values frequently obtained from current yield data analysis of 70 grapevine initial selection trials and also supported by Gonçalves et al., 2007). These errors were generated in Proc SIM2D of SAS version 9.1 (SAS Institute, 2003), 100 simulations for each field layout.

Regarding the field layouts, in many of these trials conflicts frequently arise between what is theoretically more correct and what is feasible in practice. Theoretically, the better estimates of genetic variability and a more successfully selection would be obtained from the greatest number of replicates, that is, from replicates with single plant plots. However, in practice, the management of a grapevine initial trial with thousands of plots will be very difficult, increasing the experimental errors associated to the data collection. Consequently, four replicates were adopted with four plants per plot to save on trial area needed, for greater security of plot boundaries (so that they coincided with vine trellis posts) and because often the wood of a mother plant is insufficient to make a clone with more than 16 plants. With a spacing of $1.2 \mathrm{~m} \times 2.5 \mathrm{~m}$, the conditions above produce a distance to the centre of adjacent plots of $2.5 \mathrm{~m}$ in row and $4.8 \mathrm{~m}$ in column directions.

In grapevine trials the replicates are usually contiguous, therefore latinized designs were simulated and the respective effect (latinized block or latinized column) will be included in the model for data analysis. However, according to the parsimony principle, this effect can always be discarded when it does not improve the fit of the model to the data.

Various types of experimental designs were applied to each field layout arranged by rows $((1, \ldots, r \times k)$ and columns $(1, \ldots, s)$ (Table 1$)$. The RCB design was generated using the Proc Plan of SAS version 9.1 (SAS Institute, 2003). The $\alpha$ designs, latinized by block, RC designs and spatial RC (RCSpatial) designs, latinized by column, were generated using the package CycDesigN 3.0 (Whitaker et al., 2007). In the RCSpatial design the separation of different genotypes in rows and columns was ensured according to a modified exponential variance weight function, with a value of 0.9 for the
Table 1 Field layout, experimental designs for $r=4$ resolvable replicates and other design parameters: $g-$ no. of genotypes, $s-$ no. of incomplete blocks per replicate, for $\alpha$ design, or number of columns, for row-column designs, $k$-incomplete block size, for $\alpha$ design, or number of rows, for row-column designs

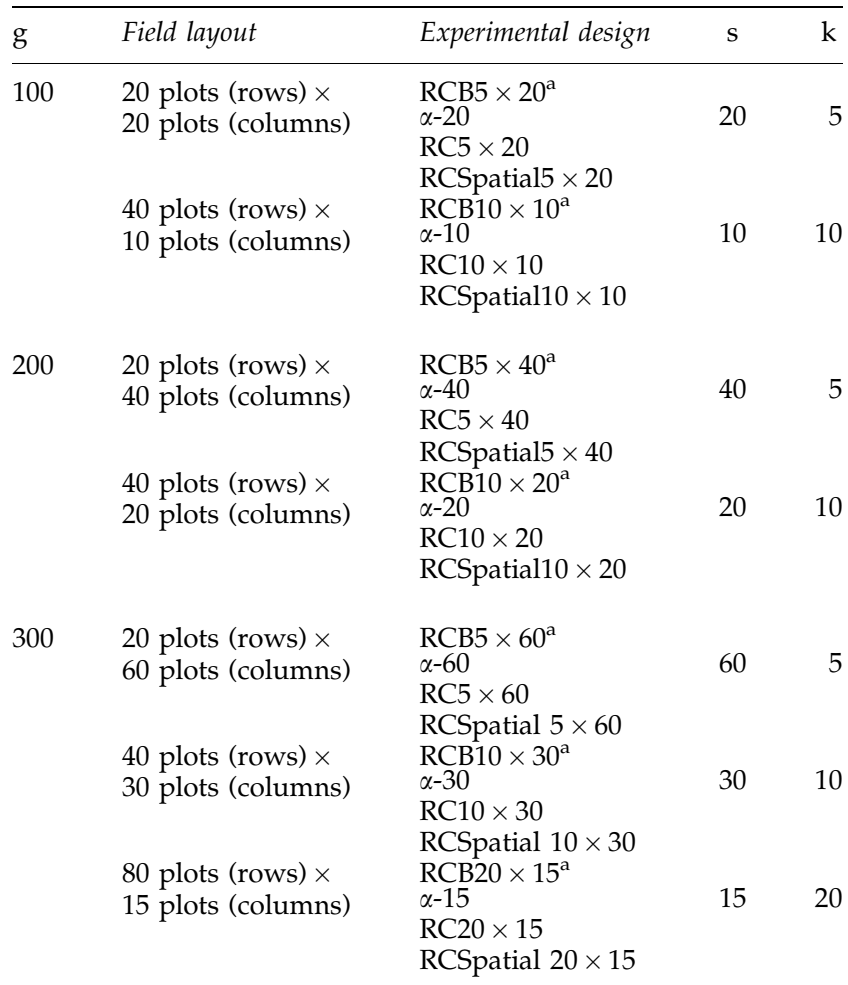

Abbreviations: RC, row-column; RCB, randomized complete block. ${ }^{\mathrm{a}}$ Rows $\times$ columns arrangement within each complete block.

decay factor, which is coherent with values from real grapevine selection trials.

For each situation described in Table 1, 100 different randomizations were done. Altogether, 11200 simulations were generated, 100 for each of 112 studied cases: population with 100 clones $\times 2$ genetic variances $\times 2$ error variances $\times 2$ field layouts $\times 4$ experimental designs (32 situations); population with 200 clones $\times 2$ genetic variances $\times 2$ error variances $\times 2$ field layouts $\times 4$ experimental designs (32 situations); population with 300 clones $\times 2$ genetic variances $\times 2$ error variances $\times 3$ field layouts $\times 4$ experimental designs (48 situations).

\section{Models for data analysis}

The linear model used for data analysis of an experiment with an RCB design was

$$
y_{i j}=\mu+u_{g_{i}}+u_{r_{j}}+e_{i j}
$$

for $i=1, \ldots, g$ and $j=1, \ldots, r$. The $y_{i j}$ represent the observations, $\mu$ the population mean, $u_{g_{i}}$ the genotypic effects, $u_{r_{j}}$ the resolvable replicate effects (complete block effects) and $e_{i j}$ the random errors associated with individual plots.

The linear model for an $\alpha$ design, latinized by block, was

$$
y_{i j t l}=\mu+u_{g_{i}}+u_{r_{j}}+u_{\text {blat }_{t}}+u_{b(r)_{j l}}+e_{i j t l}
$$


for $i=1, \ldots, g, j=1, \ldots, r, t=1, \ldots, s, \quad l=1, \ldots, s$. The $y_{i j t l}$ represent the observations, $\mu$ the population mean, $u_{g_{i}}$ the genotypic effects, $u_{r_{j}}$ the resolvable replicate effects, $u_{\text {blat }_{t}}$ the latinized block effects, $u_{b(r)_{j l}}$ the incomplete block effects within replicates and $e_{i j t l}$ the random errors associated with individual plots.

The linear model for a resolvable RC design, latinized by column, was

$y_{i j t l m}=\mu+u_{g_{i}}+u_{r_{j}}+u_{l \operatorname{col}_{t}}+u_{\operatorname{col}(r)_{j l}}+u_{\text {row }(r)_{j m}}+e_{i j t l m}$

for $i=1, \ldots, g, j=1, \ldots, r, t=1, \ldots, s, l=1, \ldots, s, m=1, \ldots, k$. The $y_{i j t l m}$ represent the observations, $\mu$ the population mean, $u_{g_{i}}$ the genotypic effects, $u_{r_{i}}$ the resolvable replicate effects, $u_{l_{\text {col }} t}$ the latinized column effects, $u_{\operatorname{col}(r)_{i l}}$ the column effects within replicates, $u_{\text {row }(r)_{i m}}$ the row effects within replicates and $e_{i j t l m}$ the random errors associated with individual plots.

In all cases, model effects (with the exception of $\mu$ ) were assumed iid normal variables with 0 mean and respective variances $\sigma_{g}^{2}, \sigma_{r}^{2}, \sigma_{\text {blat }}^{2}, \sigma_{b(r)}^{2}, \sigma_{l \mathrm{col}}^{2}, \sigma_{\mathrm{col}(r)}^{2}$, $\sigma_{\text {row }(r)}^{2}$ and $\sigma_{e}^{2}$. All random effects were assumed mutually independent.

It should be noted that, although we know the real error variance-covariance structure, and it is possible to incorporate it into models 1, 2 and 3, this was not the objective and so we considered iid errors. What one would expect is that the spatially dependent error component, which was initially simulated and incorporated into the data would now be captured, in some extent, by the effects of the design factors. A second justification for the non-fitting of spatial models was to avoid making comparisons of models that are influenced by the way the data were generated. We always followed this strategy, even for data analysis of spatial RC designs, which were analyzed as a classical $\mathrm{RC}$ design.

All models were fitted in Proc Mixed (Littell et al., 2006) of SAS version 9.1 (SAS Institute, 2003).

\section{Model parameters evaluation and effects on genetic selection}

Model parameters were estimated by the residual or restricted maximum likelihood method (REML, Patterson and Thompson, 1971), using the Fisher-scoring algorithm (Jennrich and Sampson, 1976).

To understand the fraction of the total variance accounted for by each of the design effects, the results were expressed in terms of the percentage of each component of variance resulting from each effect of the model. In addition, the relative bias (RB) and the mean squared error (MSE) of the estimates of the genotypic variance component were calculated to assess its accuracy and its precision.

To compare the effects of different experimental designs and, therefore, of the respective models for data analysis (models 1, 2 and 3) on efficiency of genetic selection, the following indicators were calculated (expressed as the average of the 100 simulations, for each case studied).

(i) For the prediction of the genotypic effects of the clones, empirical best linear unbiased predictorsEBLUPs-of genotypic effects of the clones $\left(\tilde{u}_{g}\right)$ were obtained through mixed model equations (Henderson 1975; Searle et al., 1992). (ii) For evaluating the precision in the prediction of genotypic effects, the prediction standard errors (PSEs) based on the comparison of $\tilde{u}_{g}$ with the simulated genotypic random effects were computed.

(iii) Relative efficiency (RE) of experimental design D1 compared with experimental design D2, defined as

$$
\operatorname{RE}_{\mathrm{APSE}}\left(\mathrm{D}_{1} \text { to } \mathrm{D}_{2}\right)(\%)=\frac{\mathrm{APSE}_{\mathrm{D} 2}}{\mathrm{APSE}_{\mathrm{D} 1}} \times 100
$$

where APSE is the average prediction standard error of $\tilde{u}_{g}$.

(iv) Spearman's rank correlation coefficient $\left(r_{\mathrm{s}}\right)$ and the associated standard error, for the comparison between the rankings of EBLUPs of the genotypic effects and the true genotypic effects (the simulated effects).

(v) RB and the MSE of the predicted genetic gain (PGG) ( RB $_{\text {PGG }}$ and $\mathrm{MSE}_{\mathrm{PGG}}$, respectively) for the selection of the group of 30 top-ranking clones for the populations of 100 and 200 clones, and 45 top-ranking clones for the population of 300 clones. The PGG was calculated for each simulation as the average of the EBLUPs of the top selected clones. The true genetic gain was calculated in the same way, but using genetic effects generated for each situation.

To clarify if it is possible to control the effect of the spatial autocorrelation through the experimental design, semivariograms of the errors in simulated populations were compared with those of the residuals from various fitted models (which describe the spatial correlation not accounted for by the design effects). More precisely, a simulation of two field layouts for populations with 300 genotypes was studied, and plots of the sample semivariogram (Matheron, 1963) were computed through Proc Variogram and Proc Gplot of SAS.

\section{Results}

For the level 1 of error variance, the estimates of restricted maximum likelihood of genotypic variance were close to the parametric values imposed during the simulation (that is values of 0.2025 and 0.81 ), revealing a very low RB (varying between -4 and $6 \%$ ). This occurred for all the experimental designs (including the RCB design), for any size of population (that is for 100, 200 and 300 genotypes) and for the two levels of genetic variation (Table 2). However, the MSE of the genetic variance estimates was lower (chiefly by a reduction in its variance) for $\alpha$ and RC designs, and decreased as the number of clones in the trial increased. For the level 2 of error variance similar results were obtained, but a higher $\mathrm{RB}$ and MSE associated with these estimates were observed, especially for trials with 300 clones under low genetic variability and RCB designs (Table 3 ).

The RCB design showed the greatest percentage of total variance attributable to error, coming out one percentage point lower in this component in the $\alpha$ and RC designs. Furthermore, it was observed that among the RCB designs, the closer the resolvable complete block was to the square, the greater the percentage of total variance attributable to component $\sigma_{r}^{2}$ and the less it is attributable to $\sigma_{e}^{2}$.

In Tables 2 and 3, it is clear that among $\alpha$ designs, the greater the number of incomplete blocks and the smaller their size, the greater is the percentage of total variance attributable to incomplete blocks $\left(\sigma_{b(r)}^{2}\right)$ and to latinized 
Table 2 Relative bias (RB) and mean squared error (MSE) of the genotypic variance estimate $\left(\hat{\sigma}_{g}^{2}\right)$ and fraction of the total variance estimate (FTV) attributable to each variance component, for two levels of genotypic variance and level 1 of error variance and for 100 , 200 and 300 genotypes $(g)$ (results expressed as the average of 100 simulations)

\begin{tabular}{|c|c|c|c|c|c|c|c|c|c|c|c|}
\hline \multirow[t]{2}{*}{$\mathrm{g}$} & \multirow[t]{2}{*}{ Experimental design } & \multicolumn{2}{|c|}{$\hat{\sigma}_{\mathrm{g}}^{2}$} & \multirow{2}{*}{$\begin{array}{c}\hat{\sigma}_{\mathrm{g}}^{2} \\
F T V(\%)\end{array}$} & \multirow[t]{2}{*}{$\hat{\sigma}_{\mathrm{r}}^{2}$} & \multirow[t]{2}{*}{$\hat{\sigma}_{\text {blat }}^{2}$} & \multirow[t]{2}{*}{$\hat{\sigma}_{b(r)}^{2}$} & \multirow[t]{2}{*}{$\hat{\sigma}_{\mathrm{lcol}}^{2}$} & \multirow[t]{2}{*}{$\hat{\sigma}_{r o w(\mathbf{r})}^{2}$} & \multirow[t]{2}{*}{$\hat{\sigma}_{c o l(\mathrm{r})}^{2}$} & \multirow[t]{2}{*}{$\hat{\sigma}_{\mathrm{e}}^{2}$} \\
\hline & & $R B(\%)$ & MSE & & & & & & & & \\
\hline \multicolumn{12}{|l|}{100} \\
\hline \multirow{6}{*}{ Low genetic variability } & $\mathrm{RCB} 10 \times 10$ & -1.1 & 0.006 & 13.3 & 14.7 & & & & & & 72.0 \\
\hline & $\alpha-10$ & -0.9 & 0.005 & 13.2 & 14.4 & 0.8 & 2.7 & & & & 68.9 \\
\hline & $\mathrm{RC} 10 \times 10^{\mathrm{a}}$ & 5.2 & 0.005 & 13.9 & 12.9 & & & 0.9 & 14.8 & 3.8 & 53.7 \\
\hline & RCB5 × 20 & 0.1 & 0.006 & 13.9 & 11.8 & & & & & & 74.3 \\
\hline & $\alpha-20$ & 1.2 & 0.006 & 13.9 & 11.5 & 3.3 & 8.8 & & & & 62.6 \\
\hline & $\mathrm{RC} 5 \times 20^{\mathrm{a}}$ & -2.7 & 0.004 & 13.3 & 10.3 & & & 4.2 & 5.8 & 9.4 & 57.0 \\
\hline \multirow[t]{6}{*}{ High genetic variability } & $\mathrm{RCB} 10 \times 10$ & 4.1 & 0.028 & 39.0 & 10.3 & & & & & & 50.7 \\
\hline & $\alpha-10$ & -1.2 & 0.026 & 37.6 & 10.3 & 0.9 & 2.1 & & & & 49.2 \\
\hline & $\mathrm{RC} 10 \times 10^{\mathrm{a}}$ & 1.6 & 0.028 & 38.4 & 9.2 & & & 0.6 & 10.5 & 2.8 & 38.5 \\
\hline & $\mathrm{RCB} 5 \times 20$ & 3.7 & 0.026 & 40.2 & 8.3 & & & & & & 51.5 \\
\hline & $\alpha-20$ & -1.0 & 0.024 & 38.7 & 8.1 & 2.5 & 6.0 & & & & 44.6 \\
\hline & $\mathrm{RC} 5 \times 20^{\mathrm{a}}$ & 1.9 & 0.031 & 39.5 & 7.3 & & & 2.5 & 3.8 & 6.5 & 40.4 \\
\hline \multicolumn{12}{|l|}{200} \\
\hline \multirow{6}{*}{ Low genetic variability } & $\mathrm{RCB} 10 \times 20$ & -1.2 & 0.006 & 12.8 & 13.6 & & & & & & 73.6 \\
\hline & $\alpha-20$ & 1.2 & 0.002 & 13.1 & 13.4 & 1.2 & 5.5 & & & & 66.8 \\
\hline & $\mathrm{RC} 10 \times 20^{\mathrm{a}}$ & 2.6 & 0.002 & 13.2 & 12.5 & & & 1.2 & 8.4 & 6.4 & 58.3 \\
\hline & RCB5 × 40 & 0.3 & 0.006 & 13.5 & 9.0 & & & & & & 77.5 \\
\hline & $\alpha-40$ & 3.8 & 0.002 & 13.7 & 8.6 & 4.1 & 14.1 & & & & 59.6 \\
\hline & $\mathrm{RC} 5 \times 40^{\mathrm{a}}$ & -1.7 & 0.003 & 13.0 & 8.1 & & & 4.0 & 2.6 & 14.5 & 57.8 \\
\hline \multirow{6}{*}{ High genetic variability } & RCB10 × 20 & 0.2 & 0.028 & 37.5 & 9.9 & & & & & & 52.6 \\
\hline & $\alpha-20$ & 1.5 & 0.013 & 37.7 & 9.6 & 0.8 & 4.0 & & & & 47.9 \\
\hline & $\mathrm{RC} 10 \times 20^{\mathrm{a}}$ & 0.2 & 0.015 & 37.4 & 9.1 & & & 0.9 & 5.7 & 4.6 & 42.3 \\
\hline & RCB5 $\times 40$ & 0.9 & 0.028 & 38.1 & 6.7 & & & & & & 55.2 \\
\hline & $\alpha-40$ & 2.3 & 0.015 & 38.5 & 6.1 & 2.9 & 9.9 & & & & 42.5 \\
\hline & $\mathrm{RC} 5 \times 40^{\mathrm{a}}$ & -0.3 & 0.015 & 37.7 & 5.8 & & & 3.1 & 1.9 & 10.2 & 41.3 \\
\hline \multicolumn{12}{|l|}{300} \\
\hline \multirow[t]{9}{*}{ Low genetic variability } & $\mathrm{RCB} 20 \times 15$ & -1.1 & 0.006 & 12.7 & 10.4 & & & & & & 76.9 \\
\hline & $\alpha-15$ & 0.7 & 0.002 & 13.8 & 10.9 & 0.6 & 2.4 & & & & 72.3 \\
\hline & $\mathrm{RC} 20 \times 15^{\mathrm{a}}$ & 1.3 & 0.002 & 12.8 & 9.3 & & & 0.6 & 16.1 & 2.8 & 58.4 \\
\hline & RCB10 × 30 & 1.2 & 0.006 & 13.4 & 10.8 & & & & & & 75.8 \\
\hline & $\alpha-30$ & 3.5 & 0.002 & 13.4 & 10.3 & 1.7 & 7.6 & & & & 67.0 \\
\hline & $\mathrm{RC} 10 \times 30^{\mathrm{a}}$ & 3.7 & 0.002 & 13.3 & 9.6 & & & 1.7 & 6.5 & 8.2 & 60.6 \\
\hline & RCB5 × 60 & 1.3 & 0.006 & 13.2 & 6.2 & & & & & & 80.6 \\
\hline & $\alpha-60$ & 5.3 & 0.002 & 13.5 & 6.0 & 7.1 & 14.9 & & & & 58.5 \\
\hline & $\mathrm{RC} 5 \times 60^{\mathrm{a}}$ & 1.1 & 0.001 & 13.0 & 5.6 & & & 7.0 & 2.0 & 15.3 & 57.1 \\
\hline \multirow{9}{*}{ High genetic variability } & RCB20 × 15 & -0.2 & 0.028 & 37.0 & 7.5 & & & & & & 55.5 \\
\hline & $\alpha-15$ & 1.6 & 0.008 & 37.0 & 7.6 & 0.4 & 1.5 & & & & 53.4 \\
\hline & $\mathrm{RC} 20 \times 15^{\mathrm{a}}$ & 2.8 & 0.009 & 37.4 & 6.7 & & & 0.5 & 11.4 & 2.0 & 42.1 \\
\hline & $\mathrm{RCB} 10 \times 30$ & 0.8 & 0.028 & 37.3 & 7.9 & & & & & & 54.8 \\
\hline & $\alpha-30$ & 2.1 & 0.009 & 37.6 & 7.3 & 1.3 & 5.6 & & & & 48.2 \\
\hline & $\mathrm{RC} 10 \times 30^{\mathrm{a}}$ & 0.8 & 0.007 & 37.4 & 6.9 & & & 1.3 & 4.8 & 6.0 & 43.7 \\
\hline & RCB5 × 60 & 0.5 & 0.028 & 37.3 & 4.4 & & & & & & 58.3 \\
\hline & $\alpha-60$ & 2.7 & 0.008 & 37.8 & 4.3 & 5.1 & 10.7 & & & & 42.1 \\
\hline & $\mathrm{RC} 5 \times 60^{\mathrm{a}}$ & 0.7 & 0.007 & 37.3 & 4.0 & & & 5.1 & 1.5 & 11.0 & 41.1 \\
\hline
\end{tabular}

Abbreviations: RC, row-column; RCB, randomized complete block.

${ }^{\text {a }}$ The results obtained with RCSpatial design were similar.

blocks $\left(\sigma_{\text {blat }}^{2}\right)$ and the less is attributable to the components $\sigma_{r}^{2}$ and $\sigma_{e}^{2}$. Among RC designs, it was also observed that the greater the number of rows per replicate the bigger is $\hat{\sigma}_{\text {row }(r)}^{2}$ and the smaller is $\hat{\sigma}_{r}^{2}$. On the other hand, the greater the number of columns, the greater are the estimates of the variance components $\sigma_{\mathrm{col}(r)}^{2}$ and $\sigma_{\text {lcol }}^{2}$. The greater proximity between the values of $\hat{\sigma}_{\text {row }(r)}^{2}$ and $\hat{\sigma}_{\operatorname{col}(r)}^{2}$ was observed for RC10 $\times 20$ (trial with 200 clones) and for RC10 $\times 30$ (300 clones). In these situations, a reduction in variance of error $\left(\sigma_{e}^{2}\right)$ was also observed. In addition, the results obtained with spatial and non-spatial RC designs were similar (which is expectable because the model for data analysis was the same).

From the aforementioned comments it follows that the higher the number of levels of design effects, the greater is the percentage of the total variance attributable to design effects (incomplete blocks, latinized blocks, row, column, latinized columns effects). This occurs because when random effects are assumed, reduced numbers of levels give rise to more frequent null REML estimates for the respective variance components.

For the level 1 of error variance the frequency of null estimates of genotypic variance was 0 . In situations of 
Table 3 Relative bias (RB) and mean squared error (MSE) of the genotypic variance estimate $\left(\hat{\sigma}_{g}^{2}\right)$ and fraction of the total variance estimate (FTV) attributable to each variance component, for two levels of genotypic variance and level 2 of error variance and for 100,200 and 300 genotypes $(g)$ (results expressed as the average of 100 simulations)

\begin{tabular}{|c|c|c|c|c|c|c|c|c|c|c|c|c|}
\hline \multirow[t]{2}{*}{ g } & \multirow[t]{2}{*}{ Experimental design } & \multicolumn{3}{|c|}{$\hat{\sigma}_{\mathrm{g}}^{2}$} & \multirow{2}{*}{$\begin{array}{c}\hat{\sigma}_{\mathrm{g}}^{2} \\
F T V(\%)\end{array}$} & \multirow[t]{2}{*}{$\hat{\sigma}_{\mathrm{r}}^{2}$} & \multirow[t]{2}{*}{$\hat{\sigma}_{\text {blat }}^{2}$} & \multirow[t]{2}{*}{$\hat{\sigma}_{b(\mathrm{r})}^{2}$} & \multirow[t]{2}{*}{$\hat{\sigma}_{\mathrm{Icol}}^{2}$} & \multirow[t]{2}{*}{$\hat{\sigma}_{r o w(\mathbf{r})}^{2}$} & \multirow[t]{2}{*}{$\hat{\sigma}_{c o l(\mathrm{r})}^{2}$} & \multirow[t]{2}{*}{$\hat{\sigma}_{\mathrm{e}}^{2}$} \\
\hline & & $\% \hat{\sigma}_{\mathrm{g}}^{2}=0$ & $R B(\%)$ & MSE & & & & & & & & \\
\hline \multicolumn{13}{|l|}{100} \\
\hline \multirow[t]{6}{*}{ Low genetic variability } & RCB10 $\times 10$ & 11.0 & 6.3 & 0.022 & 5.2 & 16.1 & & & & & & 78.8 \\
\hline & $\alpha-10$ & 10.0 & -1.8 & 0.021 & 4.7 & 15.7 & 0.9 & 2.9 & & & & 75.7 \\
\hline & $\mathrm{RC} 10 \times 10^{\mathrm{a}}$ & 7.0 & 13.3 & 0.021 & 5.5 & 14.2 & & & 0.9 & 16.2 & 4.2 & 59.1 \\
\hline & RCB5 × 20 & 6.0 & 7.2 & 0.026 & 5.4 & 12.9 & & & & & & 81.6 \\
\hline & $\alpha-20$ & 4.0 & 4.8 & 0.024 & 5.3 & 12.5 & 3.7 & 9.6 & & & & 68.9 \\
\hline & $\mathrm{RC} 5 \times 20^{\mathrm{a}}$ & 5.0 & -3.8 & 0.016 & 4.9 & 11.4 & & & 3.9 & 6.0 & 10.6 & 63.3 \\
\hline \multirow[t]{6}{*}{ High genetic variability } & RCB10 × 10 & 0 & 8.1 & 0.063 & 18.1 & 13.8 & & & & & & 68.0 \\
\hline & $\alpha-10$ & 0 & -3.1 & 0.062 & 16.4 & 13.7 & 0.8 & 2.8 & & & & 66.3 \\
\hline & $\mathrm{RC} 10 \times 10^{\mathrm{a}}$ & 0 & 3.5 & 0.058 & 17.4 & 12.4 & & & 0.8 & 14.1 & 3.8 & 51.6 \\
\hline & RCB5 × 20 & 0 & 7.6 & 0.060 & 18.7 & 11.1 & & & & & & 70.2 \\
\hline & $\alpha-20$ & 0 & -1.5 & 0.048 & 17.3 & 10.9 & 3.3 & 8.2 & & & & 60.4 \\
\hline & $\mathrm{RC} 5 \times 20^{\mathrm{a}}$ & 0 & 3.6 & 0.045 & 18.1 & 9.9 & & & 3.3 & 5.2 & 9.0 & 54.6 \\
\hline \multicolumn{13}{|l|}{200} \\
\hline \multirow[t]{6}{*}{ Low genetic variability } & RCB10 × 20 & 3.0 & -2.5 & 0.013 & 4.6 & 13.1 & & & & & & 82.3 \\
\hline & $\alpha-20$ & 1.0 & 9.1 & 0.011 & 5.1 & 13.2 & 1.6 & 6.5 & & & & 73.6 \\
\hline & $\mathrm{RC} 10 \times 20^{\mathrm{a}}$ & 1.0 & 8.0 & 0.011 & 5.1 & 12.2 & & & 1.6 & 9.7 & 7.6 & 63.9 \\
\hline & RCB5 × 40 & 4.0 & 12.1 & 0.013 & 5.5 & 9.0 & & & & & & 85.5 \\
\hline & $\alpha-40$ & 1.0 & 15.1 & 0.013 & 5.7 & 8.7 & 4.6 & 15.3 & & & & 65.7 \\
\hline & $\mathrm{RC} 5 \times 40^{\mathrm{a}}$ & 1.0 & 10.8 & 0.016 & 5.4 & 7.9 & & & 4.7 & 2.9 & 15.9 & 63.1 \\
\hline \multirow{6}{*}{ High genetic variability } & RCB10 × 20 & 0 & -1.4 & 0.042 & 16.2 & 11.8 & & & & & & 72.0 \\
\hline & $\alpha-20$ & 0 & 3.5 & 0.027 & 17.0 & 11.5 & 1.4 & 5.8 & & & & 64.4 \\
\hline & $\mathrm{RC} 10 \times 20^{\mathrm{a}}$ & 0 & 2.7 & 0.028 & 16.9 & 10.7 & & & 1.4 & 8.5 & 6.6 & 56.0 \\
\hline & RCB5 × 40 & 0 & 3.3 & 0.036 & 17.7 & 7.8 & & & & & & 74.5 \\
\hline & $\alpha-40$ & 0 & 5.5 & 0.037 & 17.9 & 7.6 & 3.9 & 13.3 & & & & 57.3 \\
\hline & $\mathrm{RC} 5 \times 40^{\mathrm{a}}$ & 0 & 2.1 & 0.031 & 17.4 & 7.1 & & & 4.1 & 2.6 & 13.7 & 55.2 \\
\hline 300 & RCB $20 \times 15$ & 2.0 & 11.0 & 0.009 & 5.1 & 11.5 & & & & & & 83.4 \\
\hline \multirow{8}{*}{ Low genetic variability } & $\alpha-15$ & 2.0 & 0.4 & 0.010 & 4.6 & 11.3 & 0.6 & 2.7 & & & & 80.8 \\
\hline & $\mathrm{RC} 20 \times 15^{\mathrm{a}}$ & 1.0 & 0.3 & 0.008 & 4.6 & 10.5 & & & 0.7 & 17.4 & 3.3 & 63.5 \\
\hline & RCB10 × 30 & 0 & 14.1 & 0.010 & 5.4 & 11.0 & & & & & & 83.6 \\
\hline & $\alpha-30$ & 0 & -7.6 & 0.007 & 4.3 & 10.9 & 2.6 & 8.8 & & & & 73.4 \\
\hline & $\mathrm{RC} 10 \times 30^{\mathrm{a}}$ & 0 & 4.4 & 0.006 & 4.9 & 10.1 & & & 2.7 & 7.1 & 9.4 & 65.8 \\
\hline & RCB5 × 60 & 1.0 & 2.3 & 0.008 & 4.8 & 5.7 & & & & & & 89.5 \\
\hline & $\alpha-60$ & 1.0 & 2.5 & 0.007 & 4.8 & 5.4 & 7.4 & 18.1 & & & & 64.2 \\
\hline & $\mathrm{RC} 5 \times 60^{\mathrm{a}}$ & 0 & -1.8 & 0.005 & 4.6 & 5.1 & & & 7.4 & 2.1 & 18.6 & 62.2 \\
\hline \multirow{9}{*}{ High genetic variability } & RCB20 × 15 & 0 & 2.9 & 0.022 & 16.7 & 10.1 & & & & & & 73.2 \\
\hline & $\alpha-15$ & 0 & 2.1 & 0.025 & 16.6 & 10.0 & 0.6 & 2.3 & & & & 70.5 \\
\hline & $\mathrm{RC} 20 \times 15^{\mathrm{a}}$ & 0 & 1.5 & 0.024 & 16.5 & 9.2 & & & 0.6 & 15.3 & 2.9 & 55.5 \\
\hline & RCB10 × 30 & 0 & 3.1 & 0.021 & 17.0 & 9.7 & & & & & & 73.4 \\
\hline & $\alpha-30$ & 0 & -0.1 & 0.016 & 16.4 & 9.4 & 2.3 & 7.6 & & & & 64.2 \\
\hline & $\mathrm{RC} 10 \times 30^{\mathrm{a}}$ & 0 & -0.1 & 0.017 & 16.5 & 8.8 & & & 2.3 & 6.3 & 8.4 & 57.8 \\
\hline & RCB5 $\times 60$ & 0 & 1.4 & 0.026 & 16.7 & 5.0 & & & & & & 78.3 \\
\hline & $\alpha-60$ & 0 & 1.6 & 0.020 & 16.7 & 4.8 & 6.6 & 15.8 & & & & 56.1 \\
\hline & $\mathrm{RC} 5 \times 60^{\mathrm{a}}$ & 0 & 1.6 & 0.015 & 16.7 & 4.4 & & & 6.5 & 2.0 & 16.0 & 54.3 \\
\hline
\end{tabular}

Abbreviations: RC, row-column; RCB, randomized complete block.

${ }^{a}$ The results obtained with RCSpatial design were similar.

higher error variance (level 2) and low genetic variability, the frequency of simulations, which caused null estimates of the genotypic variance component $\left(\hat{\sigma}_{g}^{2}=0\right)$, was greater in trials with 100 genotypes (6-11\%) and decreased with the rise in the number of genotypes. The choice of an $\alpha$ design or a RC design led to a reduction in null estimates of genotypic variance (Table 3).

As the level 1 of error variance is the more frequent situation, we just illustrate the results related to EBLUPs of genetic effects for this case. For the three population sizes and for the two genetic variation levels, the PSEs of EBLUPs of genotypic effects were smaller for $\alpha$ and RC designs than for RCB design (Table 4). The greatest efficiency of those designs relative to $\mathrm{RCB}$ design can be seen when working with populations with 300 clones and with higher genetic variation, thereby obtaining RE values of $108.4 \%$.

The greatest efficiency of the $\mathrm{RC}$ designs relative to the $\alpha$ designs occurs when the number of plots per incomplete block is greater or equal to 10 . In other words, for populations of 100 genotypes, the efficiency of the $\mathrm{RC} 10 \times 10$ and of the RCSpatial10 $\times 10$ is greater relative to the $\alpha-10$, for populations of 200 genotypes the efficiency of the RC10 $\times 20$ and RCSpatial10 $\times 20$ is 
Table 4 Average prediction standard error (APSE) of EBLUPs of genotypic effects and relative efficiency (RE) for populations with 100, 200 and 300 genotypes $(g)$ and level 1 of error variance

\begin{tabular}{|c|c|c|c|c|c|c|c|}
\hline \multirow[t]{2}{*}{ g } & \multirow[t]{2}{*}{ Experimental design } & \multicolumn{3}{|c|}{ Low genetic variability } & \multicolumn{3}{|c|}{ High genetic variability } \\
\hline & & APSE & $R E(\%)^{\mathrm{a}}$ & $R E(\%)^{\mathrm{b}}$ & APSE & $R E(\%)^{\mathrm{a}}$ & $R E(\%)^{\mathrm{b}}$ \\
\hline \multirow[t]{8}{*}{100} & RCB5 $\times 20$ & 0.1402 & & & 0.1839 & & \\
\hline & $\alpha-20$ & 0.1376 & 101.8 & & 0.1814 & 101.4 & \\
\hline & RC5 $\times 20$ & 0.1356 & 103.4 & 101.5 & 0.1770 & 103.9 & 102.5 \\
\hline & RCSpatial5 $\times 20$ & 0.1359 & 103.1 & 101.3 & 0.1777 & 103.5 & 102.1 \\
\hline & RCB10 × 10 & 0.1416 & & & 0.1844 & & \\
\hline & $\alpha-10$ & 0.1378 & 102.8 & & 0.1843 & 100.0 & \\
\hline & $\mathrm{RC} 10 \times 10$ & 0.1344 & 105.4 & 102.5 & 0.1758 & 104.9 & 104.9 \\
\hline & RCSpatial $10 \times 10$ & 0.1334 & 106.1 & 103.3 & 0.1777 & 103.8 & 103.8 \\
\hline \multirow[t]{8}{*}{200} & RCB5 $\times 40$ & 0.1407 & & & 0.1892 & & \\
\hline & $\alpha-40$ & 0.1366 & 103.0 & & 0.1814 & 104.3 & \\
\hline & $\mathrm{RC} 5 \times 40$ & 0.1352 & 104.0 & 101.0 & 0.1791 & 105.7 & 101.3 \\
\hline & RCSpatial5 $\times 40$ & 0.1359 & 103.5 & 100.5 & 0.1805 & 104.8 & 100.5 \\
\hline & RCB10 × 20 & 0.1398 & & & 0.1880 & & \\
\hline & $\alpha-20$ & 0.1379 & 101.3 & & 0.1842 & 102.0 & \\
\hline & $\mathrm{RC} 10 \times 20$ & 0.1350 & 103.6 & 102.2 & 0.1793 & 104.9 & 102.7 \\
\hline & RCSpatial10 $\times 20$ & 0.1361 & 102.7 & 101.4 & 0.1789 & 105.1 & 103.0 \\
\hline \multirow[t]{12}{*}{300} & RCB5 $\times 60$ & 0.1423 & & & 0.1933 & & \\
\hline & $\alpha-60$ & 0.1370 & 103.9 & & 0.1810 & 106.8 & \\
\hline & RC5 $\times 60$ & 0.1360 & 104.6 & 100.7 & 0.1789 & 108.1 & 101.2 \\
\hline & RCSpatial $5 \times 60$ & 0.1369 & 104.0 & 100.1 & 0.1783 & 108.4 & 101.5 \\
\hline & RCB10 × 30 & 0.1406 & & & 0.1891 & & \\
\hline & $\alpha-30$ & 0.1388 & 101.3 & & 0.1838 & 102.9 & \\
\hline & $\mathrm{RC} 10 \times 30$ & 0.1373 & 102.4 & 101.1 & 0.1812 & 104.3 & 101.4 \\
\hline & RCSpatial10 × 30 & 0.1374 & 102.4 & 101.0 & 0.1789 & 105.7 & 102.7 \\
\hline & RCB20 × 15 & 0.1412 & & & 0.1899 & & \\
\hline & $\alpha-15$ & 0.1404 & 100.5 & & 0.1880 & 101.0 & \\
\hline & $\mathrm{RC} 20 \times 15$ & 0.1360 & 103.8 & 103.3 & 0.1768 & 107.4 & 106.4 \\
\hline & RCSpatial20 × 15 & 0.1349 & 104.7 & 104.1 & 0.1754 & 108.2 & 107.2 \\
\hline
\end{tabular}

Abbreviations: EBLUPs, empirical best linear unbiased predictors; RC, row-column; RCB, randomized complete block.

${ }^{a}$ Relative efficiency of $\alpha$ and row-column designs compared with RCB design.

${ }^{b}$ Relative efficiency of row-column designs compared with $\alpha$ design.

greater relative to the $\alpha-20$ and for populations of 300 genotypes the efficiency of the RC20 $\times 15$ and the RCSpatial $20 \times 15$ is greater relative to the $\alpha-15$. In the latter case, the RE of RC relative to the $\alpha$ designs is in the region of $107 \%$, for populations with higher genetic variation (Table 4).

Comparing the rankings of EBLUPs of the genotypic effects and the true genotypic effects, Spearman's rank correlation coefficient values with lower standard error were obtained for the $\alpha$ and RC designs (Table 5).

As expected, all these results are reflected in the RB and in the MSE of genetic gains obtained with the selection of a group of clones (genotypic mass selection). With the $\alpha$ designs, smaller $\mathrm{RB}_{\mathrm{PGG}}$ and MSE $\mathrm{PGG}_{\mathrm{PG}}$ were obtained as the number of plots per incomplete block decreased, confirming once more that smaller incomplete blocks are more efficient for controlling environmental variation. In contrast, when the number of plots per column increased, smaller values for the $\mathrm{RB}_{\text {PGG }}$ and MSE $_{\text {PGG }}$ were observed with the RC designs, mainly for trials with 200 and 300 clones (Table 5). On the basis of these results it is easy to see how much can be gained in precision when working with populations with high genetic variation. An RB of around $-13 \%$ can be observed with the $\alpha$ and RC designs (that is much closer to the true genetic gain), whereas with low variation, this $\mathrm{RB}$ is in the region of $-33 \%$.

\section{Discussion}

As for the estimates of the component of genotypic variance (quantification of the yield genetic variability in a population), $\alpha$ and RC designs were observed to be more beneficial than RCB design (estimates with less variance), especially when the number of clones increased. However, no single design was entirely inefficient for quantification of genetic variability. These results were predictable as we are working with large samples of genotypes (100, 200 and 300). In fact, when trying to obtain an estimate of the genetic parameter relating to a group of genotypes (as in this case of genotypic variance), individual deviations are minimized by increasing the number of genotypes and the final estimate is reliable, even with a certain amount of environmental variation. However, the same conclusion cannot be reached with regard to the accuracy of the mass genotypic selection. This is explicable if we consider that making selection involves taking decisions (acceptance or rejection) regarding each individual genotype, so that the presence of a large experimental error may lead to wrong decisions. With the RC designs 
Table 5 Spearman's rank correlation coefficient $\left(r_{\mathrm{s}}\right)$ and associated standard error (s.e.), predicted genetic gain (kg per plant) (PGG) and the corresponding relative bias ( $\mathrm{RB}_{\mathrm{PGG}}$ ) and mean squared error (MSE $\mathrm{PGG}$ ) for populations with 100, 200 and 300 genotypes $(g)$ and level 1 of error variance

\begin{tabular}{|c|c|c|c|c|c|c|c|c|c|}
\hline \multirow[t]{3}{*}{ g } & \multirow[t]{3}{*}{ Experimental design } & \multicolumn{4}{|c|}{ Low genetic variability } & \multicolumn{4}{|c|}{ High genetic variability } \\
\hline & & \multirow[t]{2}{*}{$\mathrm{r}_{\mathrm{s}}$ (s.e.) } & \multicolumn{3}{|c|}{ Mass genotypic selection } & \multirow[t]{2}{*}{$r_{s}$ (s.e.) } & \multicolumn{3}{|c|}{ Mass genotypic selection } \\
\hline & & & PGG & $R B_{P G G}$ & $M S E_{P G G}$ & & $P G G$ & $R B_{P G G}$ & $M S E_{P G G}$ \\
\hline \multirow[t]{8}{*}{100} & RCB5 $\times 20$ & $0.62(0.0078)$ & 0.334 & -34.7 & 0.043 & $0.85(0.0037)$ & 0.909 & -13.2 & 0.030 \\
\hline & $\alpha-20$ & $0.64(0.0068)$ & 0.342 & -33.0 & 0.039 & $0.85(0.0037)$ & 0.897 & -14.3 & 0.036 \\
\hline & $\mathrm{RC} 5 \times 20$ & $0.65(0.0065)$ & 0.339 & -33.7 & 0.037 & $0.86(0.0033)$ & 0.920 & -12.1 & 0.033 \\
\hline & RCSpatial5 $\times 20$ & $0.65(0.0065)$ & 0.344 & -32.6 & 0.037 & $0.86(0.0034)$ & 0.914 & -12.7 & 0.030 \\
\hline & $\mathrm{RCB} 10 \times 10$ & $0.62(0.0070)$ & 0.329 & -35.6 & 0.044 & $0.85(0.0036)$ & 0.910 & -13.1 & 0.031 \\
\hline & $\alpha-10$ & $0.64(0.0066)$ & 0.333 & -35.0 & 0.042 & $0.85(0.0031)$ & 0.890 & -15.4 & 0.039 \\
\hline & $\mathrm{RC} 10 \times 10$ & $0.66(0.0061)$ & 0.362 & -29.2 & 0.031 & $0.86(0.0031)$ & 0.919 & -12.2 & 0.039 \\
\hline & RCSpatial10 × 10 & $0.66(0.0068)$ & 0.372 & -27.1 & 0.029 & $0.86(0.0033)$ & 0.899 & -14.1 & 0.037 \\
\hline \multirow[t]{8}{*}{200} & RCB5 $\times 40$ & $0.62(0.0049)$ & 0.438 & -36.6 & 0.075 & $0.84(0.0033)$ & 1.188 & -13.4 & 0.051 \\
\hline & $\alpha-40$ & $0.64(0.0040)$ & 0.473 & -31.6 & 0.055 & $0.86(0.0030)$ & 1.224 & -10.8 & 0.033 \\
\hline & $\mathrm{RC} 5 \times 40$ & $0.65(0.0047)$ & 0.459 & -33.6 & 0.063 & $0.86(0.0027)$ & 1.203 & -12.3 & 0.044 \\
\hline & RCSpatial5 $\times 40$ & $0.65(0.0041)$ & 0.468 & -32.3 & 0.058 & $0.86(0.0027)$ & 1.219 & -11.1 & 0.041 \\
\hline & RCB10 × 20 & $0.62(0.0051)$ & 0.436 & -37.0 & 0.076 & $0.85(0.0032)$ & 1.189 & -13.4 & 0.052 \\
\hline & $\alpha-20$ & $0.64(0.0046)$ & 0.457 & -34.0 & 0.063 & $0.85(0.0031)$ & 1.206 & -12.1 & 0.043 \\
\hline & $\mathrm{RC} 10 \times 20$ & $0.65(0.0043)$ & 0.470 & -32.1 & 0.055 & $0.86(0.0029)$ & 1.205 & -12.2 & 0.046 \\
\hline & RCSpatial10 × 20 & $0.65(0.0048)$ & 0.450 & -34.9 & 0.065 & $0.86(0.0026)$ & 1.207 & -12.1 & 0.043 \\
\hline \multirow[t]{12}{*}{300} & RCB5 $\times 60$ & $0.61(0.0048)$ & 0.438 & -37.9 & 0.076 & $0.83(0.0029)$ & 1.184 & -15.7 & 0.061 \\
\hline & $\alpha-60$ & $0.64(0.0038)$ & 0.475 & -32.6 & 0.060 & $0.86(0.0018)$ & 1.227 & -12.6 & 0.040 \\
\hline & $\mathrm{RC} 5 \times 60$ & $0.65(0.0041)$ & 0.459 & -34.9 & 0.064 & $0.86(0.0017)$ & 1.213 & -13.6 & 0.044 \\
\hline & RCSpatial5 $\times 60$ & $0.64(0.0044)$ & 0.450 & -36.1 & 0.070 & $0.86(0.0020)$ & 1.209 & -13.9 & 0.045 \\
\hline & $\mathrm{RCB} 10 \times 30$ & $0.62(0.0048)$ & 0.446 & -36.8 & 0.073 & $0.84(0.0024)$ & 1.191 & -15.2 & 0.055 \\
\hline & $\alpha-30$ & $0.63(0.0044)$ & 0.463 & -34.3 & 0.064 & $0.85(0.0021)$ & 1.208 & -14.0 & 0.049 \\
\hline & $\mathrm{RC} 10 \times 30$ & $0.64(0.0047)$ & 0.473 & -32.9 & 0.060 & $0.85(0.0018)$ & 1.212 & -13.7 & 0.045 \\
\hline & RCSpatial10 × 30 & $0.64(0.0038)$ & 0.452 & -35.8 & 0.071 & $0.86(0.0019)$ & 1.217 & -13.3 & 0.043 \\
\hline & RCB20 × 15 & $0.61(0.0045)$ & 0.434 & -38.4 & 0.079 & $0.84(0.0025)$ & 1.186 & -15.6 & 0.058 \\
\hline & $\alpha-15$ & $0.61(0.0044)$ & 0.442 & -37.3 & 0.076 & $0.84(0.0021)$ & 1.199 & -14.6 & 0.050 \\
\hline & $\mathrm{RC} 20 \times 15$ & $0.65(0.0041)$ & 0.471 & -33.2 & 0.061 & $0.86(0.0018)$ & 1.234 & -12.1 & 0.038 \\
\hline & RCSpatial20 × 15 & $0.65(0.0038)$ & 0.463 & -34.3 & 0.064 & $0.86(0.0013)$ & 1.221 & -13.1 & 0.043 \\
\hline
\end{tabular}

Abbreviations: RC, row-column; RCB, randomized complete block.

(spatial and non-spatial) lower PSEs of EBLUPs of the genotypic effects and higher values for $r_{s}$ with lower standard error were obtained, thus these results indicate that with these experimental designs more accurate selections can be made.

Spatial autocorrelation was simulated, as the problem of spatial variability has had a major effect on our grapevine selection trials. These effects are unpredictable over the course of a trial, various natural phenomena cause this spatial correlation (fertility, water and other environmental factors) and, so are more difficult to control for at the experimental design stage. Thus, the challenge was to identify which type of experimental design will best control the type of spatial autocorrelation usually observed in large experimental populations of grapevine clones.

From the results obtained, it is clear that the component of spatially dependent error (present in the data but not modelled through the error structure) was partially absorbed by variance components associated with design factors: $\sigma_{b(r)}^{2}$ and $\sigma_{\text {blat }}^{2}$, in the case of the $\alpha$ designs, and $\sigma_{\mathrm{row}(r)}^{2}, \sigma_{\mathrm{col}(r)}^{2}$ and $\sigma_{\mathrm{lcol}}^{2}$, in the case of the RC designs. However, this result raises the question: will we be able to control the effect of the spatial autocorrelation through the experimental design?

From the observation of Figures 1 and 2, it was clear that residuals resulting from the RCB model fit showed the same pattern of spatial variation as the simulated errors. However, this correlation starts to weaken with the adjustment of the models corresponding to the $\alpha$ and RC designs, in the case of $\alpha-60, R C 5 \times 60$ and RCSpatial $5 \times 60$ (Figures $1 \mathrm{c}-\mathrm{e}$, respectively), and to the RC designs, in cases RC20 $\times 15$ and RCSpatial20 $\times 15$ (Figures $2 \mathrm{~d}$ and $\mathrm{e}$, respectively). Hence, these results support the ability of the latter designs to control the spatial correlation.

From this simulation study, it is also possible to state that if we have field layouts allowing squared replicates with 10 or more plots per row and column, we should opt for a RC design. If the field layout allows only small-scale incomplete blocks (around five plots), we could opt for an $\alpha$ design. As for the best size of incomplete block, similar conclusions have been obtained in trials on other crops (Patterson and Hunter, 1983; Fu et al., 1998; Fu et al., 1999; Gezan et al., 2006), suggesting incomplete blocks of around five plots for $\alpha$ design.

The comparative efficiency of $\alpha$ and RC designs relative to RCB design varied between 101 and $108 \%$ (Table 4). No direct comparison can be made of these values with others obtained in field experiments with other crops, as the PSE of the genotypic effects is not always used as a criterion of comparison between experimental designs. For example, if the RE was calculated, not based on PSE, but on prediction error variance, those values would be higher. However, it is worth pointing out that the biggest differences in 

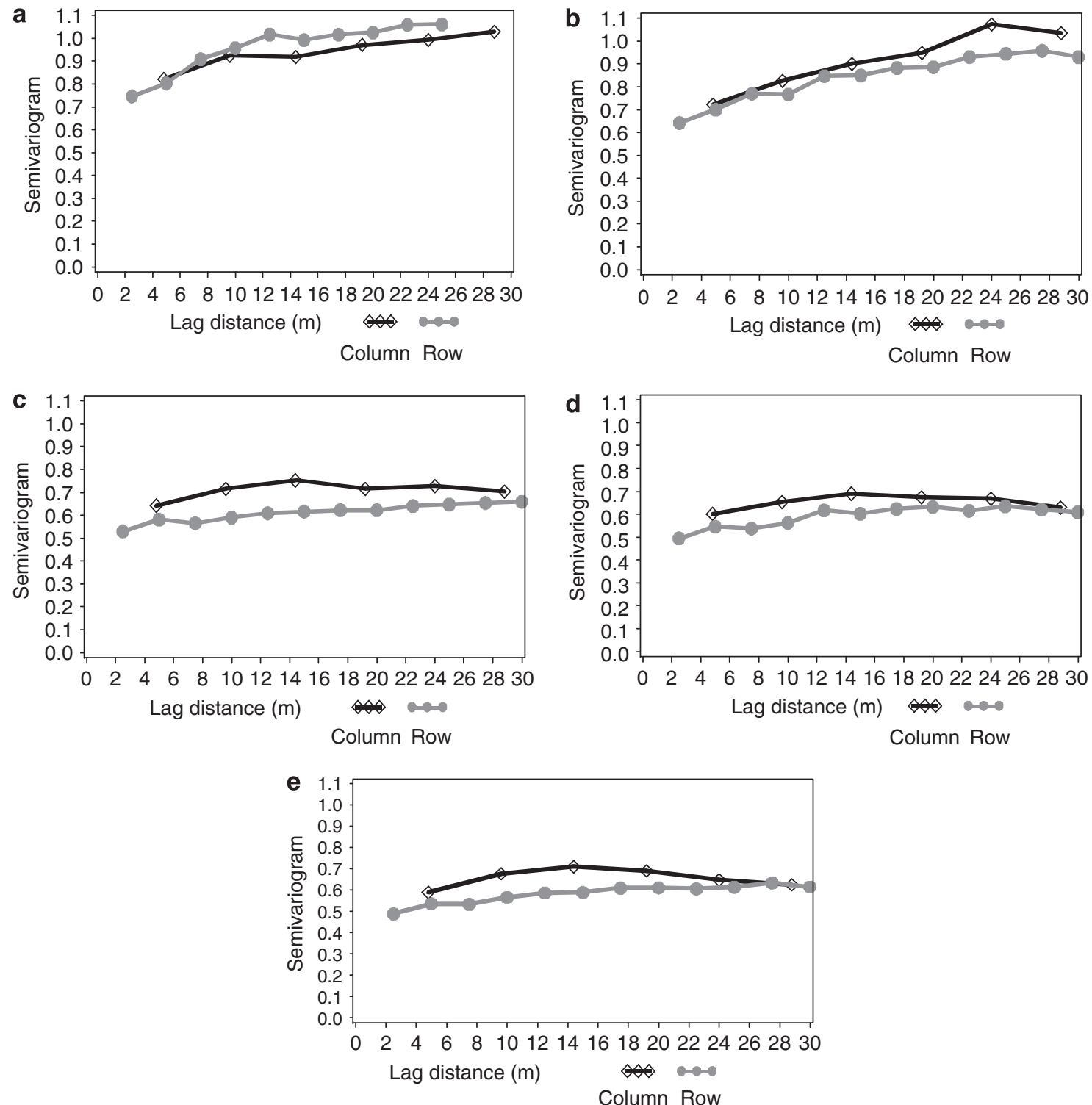

Figure 1 Empirical semivariograms for row and column directions from one simulation of a population with high genetic variability containing 300 genotypes and a field layout 20 plots (rows) $\times 60$ plots (columns): (a) simulated errors, (b) model 1 fit residuals (RCB5 $\times 60$ design), (c) model 2 fit residuals ( $\alpha-60$ design), (d) model 3 fit residuals (RC5 $\times 60$ design), (e) model 3 fit residuals (RCSpatial5 $\times 60$ design).

efficiency among experimental designs will be found whenever the size of the trial is large (higher number of genotypes) and when there is more environmental heterogeneity. Hence, as environmental variation is never entirely predictable at the beginning of a field trial, we should be ambitious in our choice of experimental design for large trials of grapevine clones, always opting for $\alpha$ or resolvable RC designs, in preference to an RCB design. Besides, a point in favour of those more complex resolvable designs is that there is always the option of analysing them as an RCB design.

We should also remember that an experimental design supposed entirely suitable might later prove to be inefficient in controlling for spatial variation. That is why recourse to spatial models for data analysis is another important option for the selection success (Cullis and Gleeson, 1991; Federer, 1998; Qiao et al., 2000; Dutkowski et al., 2006; Gonçalves et al., 2007). It is important to emphasize, however, that even using spatial analysis, a good experimental design is always essential. In sum, the correct strategy should be starting with a classical incomplete block or RC model and then check if addition of a spatial error model can improve the fit (note that the option for a spatial design clearly supports this sequential fitting process). This approach also defended by other authors (Williams et al., 2006; Piepho et al., 2008) is the most adequate for data analysis of large grapevine field trials. In fact, these are perennial plants, and experimenting on them is costly and prolonged, and experimental failure is very hard to bear. Therefore, demanding statistical methods are entirely justified.

Finally, we should note that the conclusions of this work represent foundations for a new knowledge on genetic variability within grapevine varieties, allowing novel strategies for genetic resources conservation as well as for selection with high genetic gains. 

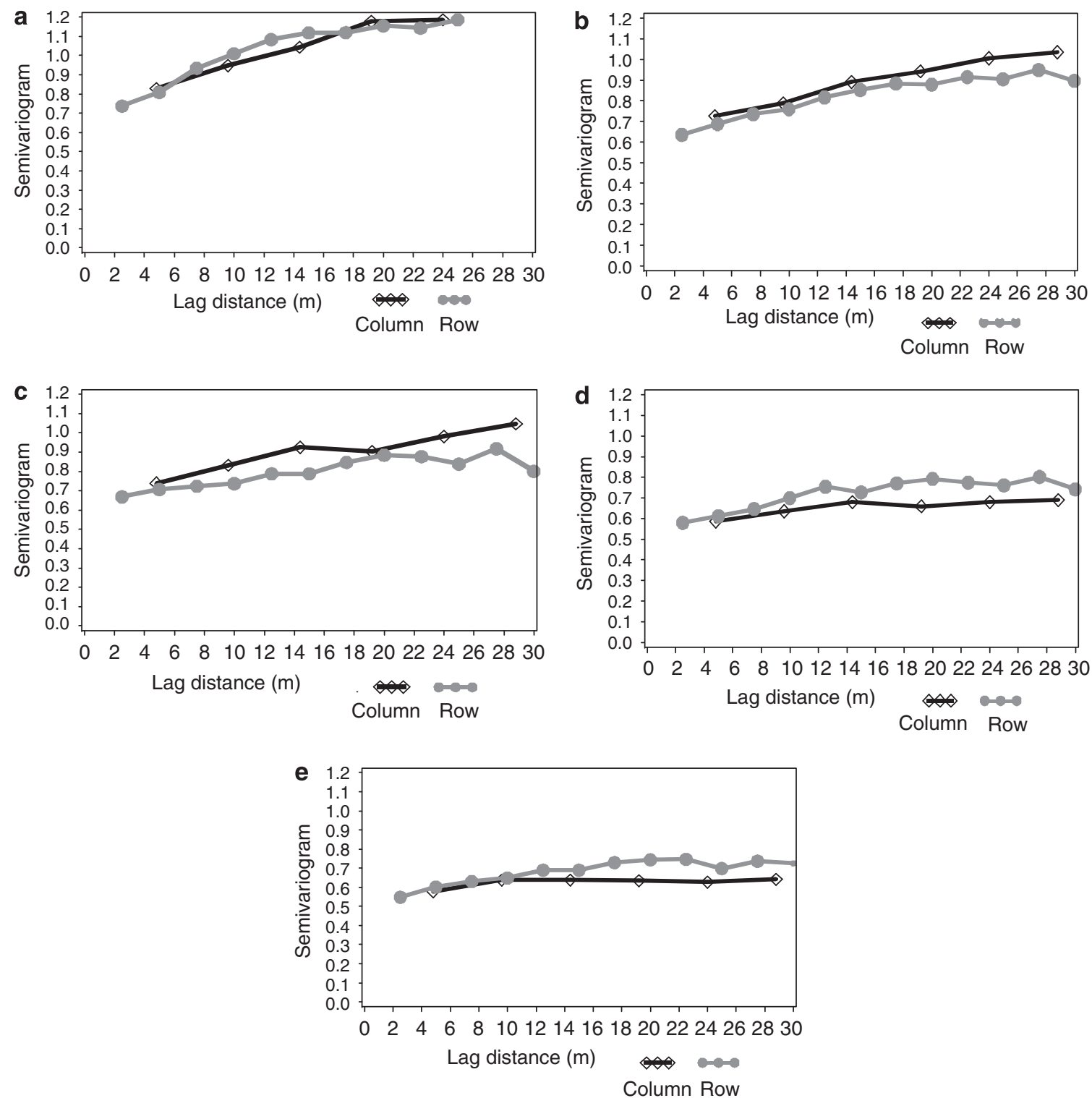

Figure 2 Empirical semivariograms for row and column directions from one simulation of a population with high genetic variability containing 300 genotypes and a field layout 80 plots (rows) $\times 15$ plots (columns): (a) simulated errors, (b) model 1 fit residuals (RCB20 $\times 15$ design), (c) model 2 fit residuals $(\alpha-15$ design), (d) model 3 fit residuals (RC20 $\times 15$ design), (e) model 3 fit residuals (RCSpatial20 $\times 15$ design).

\section{Conflict of interest}

The authors declare no conflict of interest.

\section{Acknowledgements}

We thank the 'Fundação para a Ciência e Tecnologia, Portugal' for financial support for first author. We also thank the reviewers and the editors for their valuable comments and suggestions.

\section{References}

Cullis BR, Gleeson AC (1991). Spatial analysis of field experiments-an extension to two dimensions. Biometrics 47: 1449-1460.
Dutkowski GW, Costa e Silva J, Gilmour AR, Wellendorf H, Aguiar A (2006). Spatial analysis enhances modelling of a wide variety of traits in forest genetic trials. Can J For Res 36 : 1851-1870.

Federer WT (1998). Recovery of interblock, intergradient, and intervariety information in incomplete block and lattice rectangle designed experiments. Biometrics 54: 471-481.

Fisher RA (1935). The Design of Experiments. Oliver and Boyd: Edinburgh.

Fu YB, Clarke PY, Yanchuk AD (1998). Incomplete block designs for genetic testing: statistical efficiencies of estimating family means. Can J For Res 28: 977-986.

Fu YB, Yanchuk AD, Namkoong G (1999). Incomplete block designs for genetic testing: some practical considerations. Can J For Res 29: 1871-1878.

Gezan SA, White TL, Huber DA (2006). Comparison of experimental designs for clonal forestry using simulated data. For Sci 52: 108-116. 
Gonçalves E, St.Aubyn A, Martins A (2007). Mixed spatial models for data analysis of yield on large grapevine selection field trials. Theor Appl Genet 115: 653-663.

Henderson CR (1975). Best linear unbiased estimation and prediction under a selection model. Biometrics 31: 423-447.

Jennrich RI, Sampson PF (1976). Newton-Raphson and related algorithms for maximum likelihood variance component estimation. Technometrics 18: 11-17.

John JA, Whitaker D (2000). Recursive formulae for the average efficiency factor in block and row-column designs. $J R$ Stat Soc B 62: 575-583.

John JA, Williams ER (1998). t-Latinized designs. Aust N Z J Stat 40: $111-118$

Kempton R, Seraphin J, Sword A (1994). Statistical analysis of two-dimensional variation in variety yield trials. J Agric Sci 122: 335-342.

Littell RC, Milliken GA, Stroup WW, Wolfinger RD, Schabenberger O (2006). SAS System for Mixed Models, 2nd edn. SAS Institute: Cary, NC.

Martins A (2007). Variabilidade genética intravarietal das castas. In: Böhm J (ed). Portugal vitícola, o grande livro das castas. Chaves Ferreira Publicações: Lisboa. pp 53-56.

Matheron G (1963). Principles of geostatistics. Econ Geol 58: 1246-1266.

Nguyen NK (1994). Construction of optimal block designs by computer. Technometrics 36: 300-307.

Nguyen NK (1997). Construction of optimal row-column designs by computer. Comput Sci Stat 28: 471-475.

OIV (1991). Résolution VITI 1/91, programme type pour la réalisation de la sélection clonale de la vigne. Bulletin de l'OIV 1991: 752-763.

Patterson HD, Hunter EA (1983). The efficiency of incomplete block designs in national list and recommended list cereal variety trials. J Agric Sci 101: 427-433.
Patterson HD, Silvey V (1980). Statutory and recommended list trials of crop varieties in the United Kingdom. J $R$ Stat Soc A 143: 219-252.

Patterson HD, Williams ER (1976). A new class of resolvable incomplete block designs. Biometrika 63: 83-92.

Patterson HD, Thompson R (1971). Recovery of inter-block information when block sizes are unequal. Biometrika 58: 545-554.

Patterson HD, Williams ER, Hunter EA (1978). Block designs for variety trials. J Agric Sci 90: 395-400.

Piepho HP, Richter C, Williams E (2008). Nearest neighbour adjustment and linear variance models in plant breeding trials. Biom J 50: 164-189.

Qiao CG, Basford KE, DeLacy IH, Cooper M (2000). Evaluation of experimental designs and spatial analyses in wheat breeding trials. Theor Appl Genet 100: 9-16.

SAS Institute (2003). SAS Proprietary Software. Release 9.1. SAS Institute Inc.: Cary, NC.

Searle SR, Casella G, McCulloch CE (1992). Variance Components. John Wiley \& Sons: New York.

Whitaker D, Williams ER, John JA (2007). CycDesigN: a package for computer generation of experimental designs. http:// www.cycdesign.co.nz.

Williams ER, John JA (1989). Construction of row and column designs with contiguous replicates. Appl Stat 38: 149-154.

Williams ER, John JA, Whitaker D (2006). Construction of resolvable spatial row-column designs. Biometrics 62: 103-108.

Williams ER (1985). A criterion for the construction of optimal neighbour designs. J R Stat Soc B 47: 489-497.

Yates F (1936). A new method of arranging variety trials involving a large number of varieties. J Agric Sci 26: 424-455.

Yates F (1940). Lattice squares. J Agric Sci 30: 672-687.

Yau S (1997). Efficiency of alpha-lattice designs in international variety yield trials of barley and wheat. J Agric Sci 128: 5-9. 